\title{
Estresse visual associado à dificuldade de leitura: prevalência e intervenção em alunos do ensino fundamental
}

Visual stress associated with reading difficulties: prevalence and intervention in elementary school students

Estrés visual asociado a dificultades lectoras: prevalencia e intervención en estudiantes de primaria

Recebido: 26/08/2021 | Revisado: 01/09/2021 | Aceito: 02/09/2021 | Publicado: 05/09/2021

Daniela Maggioni Pereira Leão

ORCID: https://orcid.org/0000-0001-5240-2640

Universidade Federal de Alfenas, Brasil Hospital de Olhos de Minas Gerais - Dr. Ricardo Guimarães, Brasil E-mail: dmaggioni@bol.com.br

Douglas de Araújo Vilhena

ORCID: https://orcid.org/0000-0003-2670-7963 Hospital de Olhos de Minas Gerais - Dr. Ricardo Guimarães, Brasil

Universidade Federal de Minas Gerais, Brasil

Universidade do Porto, Portugal

E-mail: lapan.douglas@gmail.com

Priscila Cardoso Ottoni

ORCID: https://orcid.org/0000-0003-1792-8094

Universidade Federal de Alfenas, Brasil

E-mail: priscillaottoni@hotmail.com

Mariana Raposo Batista

ORCID: https://orcid.org/0000-0001-7735-4518

Universidade Federal de Alfenas, Brasil

E-mail: dmarianaraposobatista@gmail.com

João Paulo Pereira Leão

ORCID: https://orcid.org/0000-0001-7630-5909

Centro Universitário Serra dos Órgãos, Brasil

E-mail: jp.sp2008@ hotmail.com

Márcia Reis Guimarães

ORCID: https://orcid.org/0000-0002-5475-5375

Hospital de Olhos de Minas Gerais - Dr. Ricardo Guimarães, Brasil

E-mail: marciag2020@gmail.com

Silvia Graciela Ruginsk

ORCID: https://orcid.org/0000-0002-0534-0077 Universidade Federal de Alfenas, Brasil E-mail: silvia.leitao@unifal-mg.edu.br

\section{Resumo}

O estresse visual é definido como uma disfunção do processamento visual, de base neuronal, com um déficit do sistema magnocelular e no córtex visual primário. Ele é caracterizado por distorções visuoperceptuais e desconforto visual progressivo na leitura. O presente estudo verificou a prevalência do estresse visual entre escolares do $3^{\circ}$ e $4^{\circ}$ ano do ensino fundamental ( 7 a 10 anos) com dificuldade de leitura, assim como investigou o efeito do uso de lâminas espectrais na habilidade de leitura. Todos os participantes elegíveis $(n=58)$ apresentavam dificuldade de leitura e adequada acuidade visual binocular. As lâminas espectrais foram selecionadas e distribuídas aos alunos para uso por três meses. A Escala de Percepção Visual de Leitura, o Teste de Taxa de Leitura e o Teste de Leitura - Compreensão e Sentença caracterizaram as condições pré e pós-intervenção. Os resultados indicaram prevalência de $12 \%$ de estresse visual (sintomas severos). Após a intervenção, foi verificado que o uso das lâminas espectrais promoveu incremento da compreensão de sentenças e da taxa de leitura. Assim, conclui-se que o estresse visual é uma disfunção muito prevalente entre crianças com dificuldade de leitura e que as lâminas espectrais são um método eficaz de intervenção para reduzir o estresse visual na leitura.

Palavras-chave: Leitura; Percepção visual; Distúrbios da visão; Criança em idade escolar; Intervenção pedagógica.

\footnotetext{
Abstract

Visual stress is defined as a neuronal-based dysfunction of visual processing, with a deficit in the magnocellular system and in the primary visual cortex. It is characterized by visual-perceptual distortions and progressive visual discomfort in reading. The present study verified the prevalence of visual stress among students from 3 rd and 4th
} 
grades of elementary school ( 7 to 10 years old) with reading difficulties, as well as investigated the effect of the use of overlays on reading ability. All eligible participants $(n=58)$ had reading difficulties and adequate binocular visual acuity. Overlays were selected and distributed to students for use for three months. The Visual Reading Perception Scale, the Reading Rate Test and the Reading Test - Comprehension and Sentence characterized the pre- and postintervention conditions. The results indicated a $12 \%$ prevalence of visual stress (severe symptoms). After the intervention, it was verified that the use of spectral slides promoted an increase in sentence comprehension and reading rate. Thus, it is concluded that visual stress is a very prevalent disorder among children with reading difficulties and that overlays are an effective method of intervention to reduce visual stress in reading.

Keywords: Reading; Visual perception; Vision disorder; Schoolchildren; Pedagogical intervention.

\section{Resumen}

El estrés visual se define como una disfunción neuronal del procesamiento visual, con un déficit en el sistema magnocelular y en la corteza visual primaria. Se caracteriza por distorsiones visual-perceptivas y malestar visual progresivo en la lectura. El presente estudio verificó la prevalencia de estrés visual en estudiantes de $3^{\circ}$ y $4^{\circ}$ grados de la escuela primaria (7 a 10 años) con dificultades lectoras, además de investigar el efecto del uso de diapositivas espectrales sobre la capacidad lectora. Todos los participantes elegibles $(n=58)$ tenían dificultades para leer y una agudeza visual binocular adecuada. Se seleccionaron diapositivas espectrales y se distribuyeron a los estudiantes para su uso durante tres meses. La Escala de Percepción de Lectura Visual, la Prueba de Tasa de Lectura y la Prueba de Lectura - Comprensión y Oración caracterizaron las condiciones previas y posteriores a la intervención. Los resultados indicaron una prevalencia del 12\% de estrés visual (síntomas graves). Luego de la intervención, se verificó que el uso de diapositivas espectrales promovió un aumento en la comprensión de oraciones y la tasa de lectura. Así, se concluye que el estrés visual es un trastorno muy prevalente entre los niños con dificultades lectoras y que los deslizamientos espectrales son un método eficaz de intervención para reducir el estrés visual en la lectura.

Palabras clave: Lectura; Percepción visual; Trastornos de la visión; Niño en edad escolar; Intervención pedagógica.

\section{Introdução}

A leitura é uma habilidade que envolve processos neurobiológicos complexos, com funções cognitivas interdependentes. De acordo com o modelo da Dupla Rota, o sistema de análise visual do estímulo gráfico (traços invariantes dos grafemas) precede tanto a rota fonológica quanto a rota de reconhecimento léxico-ortográfico (Coltheart et al., 2001; Schirmer, Fontoura \& Nunes, 2004; Salles, 2005; Dehaene, 2012; Piccolo \& Salles, 2013). Falhas de processamento da análise visual durante a leitura estão subjacentes a transtornos da aprendizagem, uma vez que interferem decisivamente na aquisição e manutenção de informações (Garcia, Vilhena, Guimarães, Pinheiro \& Momensohn-Santos, 2019; Rotta, Ohweiller \& Riesgo 2016; Siqueira \& Giannetti, 2011; Vilhena, Guimarães \& Guimarães, 2019; Zucoloto \& Sisto, 2002).

De uma forma geral, as dificuldades da aprendizagem têm causas multifatoriais (Alves et al., 2021; Souza \& Silva, 2021), sendo os fatores de risco a prematuridade, o baixo peso ao nascimento, complicações gestacionais ou no parto, e exposição neonatal a tabagismo e etilismo (Graziano \& Leone, 2005; Lamônica, Carlino \& Alvarenga, 2010; Ferreira et al., 2011; Siqueira \& Giannetti, 2011; Rotta, Ohweiller \& Riesgo 2016). A dificuldade de aprendizagem também pode ter origem não orgânica, extrínseca ao indivíduo, como aquelas relacionadas à defasagem da proposta pedagógica ou da capacitação do professor, bem como problemas familiares (Siqueira \& Giannetti, 2011).

O estresse visual (também referido como síndrome de Irlen) é um distúrbio do processamento visual, caracterizado por distorções visuoperceptuais e desconforto visual progressivo na leitura, que pode dificultar a aquisição e o desenvolvimento da leitura (Bernal, 2015; Bicalho et al., 2015; Chouinard et al., 2012; Evans et al., 1996; Faria, 2011; Kruk, Sumbler \& Willows, 2008; Nandakumar \& Leat, 2008; Vilhena, Guimarães, Guimarães \& Pinheiro, 2019).

Muitos dos sinais e sintomas do estresse visual se manifestam no ambiente escolar, onde são exigidas do estudante maior atenção e eficiência visual, além do contato constante com o material impresso (Costa, 2014; Wilkins \& Evans, 2010). O contraste das letras pretas no papel branco, a qualidade da impressão e o brilho provocado pela incidência da luz sobre o papel são fatores que podem levar as pessoas com estresse visual a um maior esforço para manter a atividade de leitura. Como consequência, são frequentemente relatadas distorções visuoperceptuais, como letras ou palavras que aparecem borradas, 
vibram, pulsam, mudam de posição, se movimentam (Boyle \& Jindal-Snape, 2012; Evans et al., 1995, 1996; Uccula, Enna \& Mulatti, 2014).

Os estudantes dos anos iniciais do Ensino Fundamental ainda estão consolidando a alfabetização e não se tornaram proficientes na leitura, sendo esse o período em que normalmente são identificadas as dificuldades de aprendizagem (Rotta, Ohlweiler \& Riesgo 2016; Schirmer, Fontoura \& Nunes, 2004; Zucoloto \& Sisto, 2002). A importância da identificação assertiva e precoce do estresse visual durante o desenvolvimento infantil reside no fato de que o aperfeiçoamento progressivo da visão depende da qualidade da informação captada pelos olhos e interpretada pelo encéfalo. Assim, se houver, nesse período, algum problema na aquisição e processamento visual, tudo o que posteriormente vier a se consolidar, em termos de funções cognitivas e motoras, estará comprometido (Faria, 2011; Graziano \& Leone, 2005; Oliveira et al., 2016; Rotta, Ohlweiler \& Riesgo 2016; Siqueira \& Giannetti, 2011).

Ademais, uma vez que o estresse visual frequentemente coexiste com a dificuldade de leitura, Transtorno de Déficit de Atenção/Hiperatividade e Transtorno do Espectro Autista, a identificação diferencial é imprescindível para a intervenção correta e eficaz (Vilhena, Guimarães \& Guimarães, 2019). A ausência da identificação e intervenção do estresse visual pode contribuir para um desestímulo do aluno frente à sua capacidade de aprender, afetando sua autoestima, seu interesse pelos estudos, assim como para o aumento das taxas de evasão e abandono escolar, que já são relativamente elevadas no Brasil (Auriglietti, 2014; Guimarães \& Guimarães, 2013; Silva Filho \& Araújo, 2017).

Desta forma, o presente estudo teve como objetivo central rastrear a prevalência do estresse visual entre escolares do $3^{\circ}$ e $4^{\circ}$ ano do Ensino Fundamental (7 a 10 anos) com dificuldade de leitura, de forma a fornecer um subsídio epidemiológico sobre este recorte da realidade brasileira. Além disso, o estudo de intervenção avaliou se os participantes com dificuldade de leitura em uso de lâminas espectrais por três meses melhoram a habilidade de leitura.

\section{Metodologia}

\subsection{Participantes}

Trata-se o presente trabalho de um estudo clínico intervencional, prospectivo e longitudinal (Estrela, 2018). Somente participaram da pesquisa crianças que assinaram o Termo de Assentimento e cujos pais ou responsáveis assinaram o Termo de Consentimento Livre e Esclarecido (TCLE). O estudo possui aprovação pelo Comitê de Ética em Pesquisa com Humanos da Universidade Federal de Alfenas, Minas Gerais, Brasil (CAAE 58223616.4.0000.5142, parecer n ${ }^{\circ}$ 1.817.671).

Participaram da amostra inicial 770 alunos de 4 escolas municipais de uma cidade do município de Alfenas, Minas Gerais, Brasil, sendo 3 urbanas e 1 rural. As etapas de identificação, seleção e elegibilidade para a inclusão dos participantes no estudo estão esquematizadas em fluxograma (Figura 1). Foram selecionados para a avaliação de leitura 237 alunos do $3^{\circ}$ ao $4^{\circ}$ ano do ensino fundamental com idade entre 7 a 10 anos e escolaridade.

Uma vez que erros refrativos podem comprometer a acuidade visual e a identificação do estresse visual, definiu-se como critérios de exclusão do estudo, além da não concordância de participação, a presença de miopia, hipermetropia ou astigmatismo não corrigidos em um ou em ambos os olhos. Para o estudo de prevalência, satisfizeram os critérios de elegibilidade 58 participantes com dificuldade de leitura ( $8.7 \pm 0.6$ anos de idade; $38 \%$ do sexo masculino; $67 \%$ do $4^{\circ}$ ano). Por fim, para o estudo de intervenção, foram avaliados no pós-teste 57 participantes. 
Figura 1. Fluxograma do número de participantes nas etapas de identificação, seleção, elegibilidade e inclusão para os estudos de prevalência e intervenção. Fonte: autores, 2021.

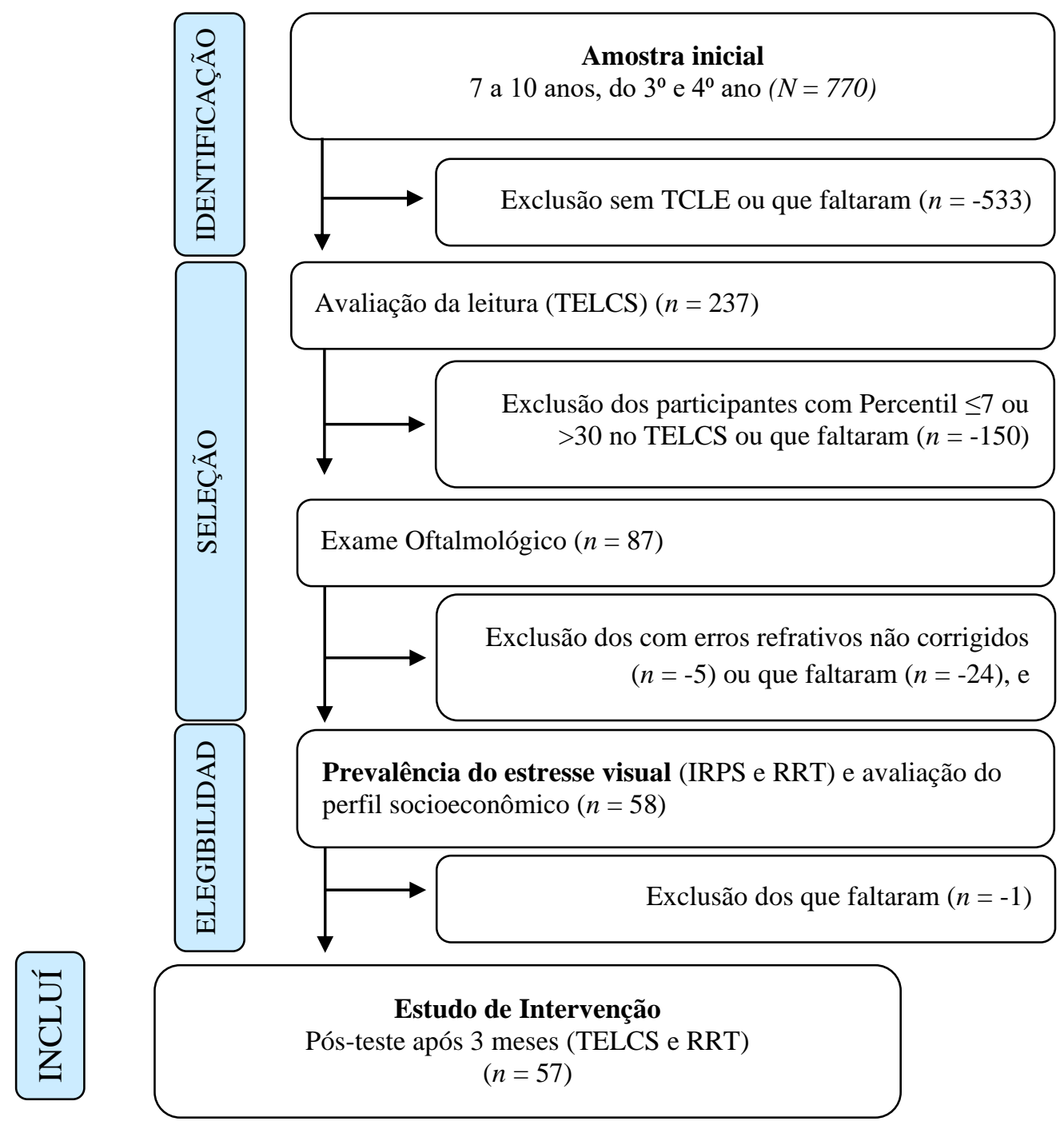

IRPS: Irlen Perceptual Reading Scale; TELCS: Teste de Leitura: Compreensão de Sentenças; RRT: Rate of Reading Test (Teste de Taxa de Leitura). Fonte: Autores (2021).

\subsection{Instrumentos}

O Teste de Leitura: Compreensão de Sentenças (TELCS) avalia a compreensão de leitura, sendo composto por 36 itens de múltipla-escolha (Vilhena, Sucena, Castro \& Pinheiro, 2016). O TELCS apresentou fontes satisfatórias de evidências de fidedignidade (alfa de Cronbach, Spearman-Brown, teste-reteste), validade de conteúdo (adaptação, controles psicolinguísticos, descrição dos itens), validade de estrutura interna (efeitos de escolaridade e idade, distribuição normal) e validade externa (convergente, discriminante, critério) (Machado \& Maluf, 2019; Medina, Souza \& Guimarães, 2018; Medina \& Guimarães, 2019, 2021; Pinheiro, Vilhena, Santos, 2017; Vilhena \& Pinheiro, 2020; Vilhena, Sucena, Castro \& Pinheiro, 2016). Vilhena e Pinheiro estabeleceram normas padronizadas para o $2^{\circ}$ ao $5^{\circ}$ ano do Ensino Fundamental (7 a 11 anos) a partir de uma amostra grande ( $n=1289$ participantes), representativa, estratificada e randomizada. O desempenho de leitura foi classificado de acordo com o Percentil: $<7=$ analfabetismo; 7 a $14=$ transtorno de leitura; 15 a $30=$ dificuldade de leitura; 31 a 59 = desempenho de leitura na média; 60 a 89 = acima da média; > 90 = superior. 
O equipamento RZ 2000 plus (Raizamed, São Paulo) foi utilizado para a análise da acuidade visual, sendo registrada a linha em que o participante conseguiu enxergar $2 / 3$ dos optotipos. Considerou-se normal a acuidade visual superior a 0,67 decimais (20/30), estabelecendo-se como déficit valores inferiores a este, baseando-se nos critérios propostos pela Organização Mundial de Saúde (Alves \& Kara-José, 1998; Toledo et al., 2010).

Para o rastreio do estresse visual e seleção das lâminas espectrais, foi utilizada a Irlen Reading Perceptual Scale (IRPS, também referida como Método Irlen), composta por quatro seções (Irlen, 2003). A Seção 1 compreende questionários sobre a dificuldade e o desconforto durante a leitura (17 itens cada), para classificar o grau em ausente ( 0 ponto), leve (1 a 3 pontos), moderado ( 4 a 7 pontos) e severo ( 8 a 17 pontos). As Seções 2,3 e 4 avaliam as respostas a diferentes tarefas que intensificam um estresse visual, sendo feita a seleção individual da lâmina espectral que oferece maior conforto para a leitura. As lâminas espectrais do Método Irlen (do inglês spectral overlays) são confeccionadas em acetato resistente e possuem um lado fosco e outro brilhante. São oferecidas comercialmente em dez tonalidades que podem ser combinadas até uma vez, o que aumenta a variedade de saturação (tons mais fortes ou mais escuros), e totaliza 65 cromaticidades (Vilhena et al., 2018).

O Teste de Taxa de Leitura (RRT) avalia o 'número de palavras lidas corretamente por minuto' e a 'porcentagem de ganho na taxa de leitura com a lâmina espectral' (Garcia, Momensohn-Santos \& Vilhena, 2017; Vilhena, Guimarães, Guimarães, \& Pinheiro, 2019). O RRT é uma adaptação para o português do Rate of Reading Test, desenvolvido por Wilkins et al. (1996). O RRT é composto por quinze vocábulos de fácil leitura, escolhidos com base nos seguintes controles linguísticos: substantivos concretos, de alta frequência de ocorrência, no singular, regulares, de curta extensão ( 2 a 4 letras), e equilibrados por gênero. Os quinze vocábulos são dispostos lado a lado em 20 linhas, com a aparência de um texto, sem relação sintática ou semântica entre si, impressos com espaçamento simples e fonte de tamanho 9.

\subsection{Procedimentos}

Para selecionar os participantes com dificuldade de leitura, aqueles que assinaram o TCLE $(n=237)$ foram submetidos coletivamente ao TELCS, com tempo limite de 5 minutos para as respostas. Não houve nenhuma criança abaixo do percentil 7 (analfabeto), sendo excluídas nesta etapa de seleção aquelas que obtiveram escore acima do percentil 30 no TELCS (bons leitores).

Os participantes com dificuldade de leitura selecionados foram submetidos a uma avaliação oftalmológica para verificar a acuidade visual, ou seja, identificar algum erro de refração (miopia, hipermetropia e astigmatismo), o qual poderia acarretar uma dificuldade de leitura. As crianças diagnosticadas com baixa acuidade visual, sem uso de lentes refrativas, foram encaminhadas para o serviço municipal de saúde.

$\mathrm{Na}$ etapa de elegibilidade, os 58 participantes que não apresentaram erros de refração na avaliação oftalmológica e as que tinham seus graus corrigidos por meio de óculos refrativos, foram submetidas ao IRPS e ao RRT para rastrear a prevalência do estresse visual e seleção das lâminas espectrais. Na administração do RRT, os participantes tiveram de ler em voz alta cada uma das cinco listas, o mais rápido possível, em um minuto, sendo o treino e os testes 2 e 3 lidos sem as lâminas espectrais, e os testes 1 e 4 lidos com o uso das lâminas espectrais (design ABBA para controle do efeito de treino). $\mathrm{O}$ exame oftalmológico e a identificação do estresse visual foram realizados pela médica oftalmologista que integra a equipe do presente estudo, sendo o desempenho de cada participante registrado em folha de respostas individuais.

Os participantes selecionados foram avaliados quanto ao perfil socioeconômico (Tabela 1), segundo o Critério de Classificação Econômica Brasil, que estima a renda domiciliar de uma família baseada em informações simples, como bens e serviços disponíveis no domicílio e a escolaridade do chefe da família (ABEP, 2016; Alves, Soares \& Xavier, 2014; Kamakura \& Mazzon, 2016). Os 58 participantes elegíveis para o estudo de prevalência e intervenção receberam uma pasta contendo a lâmina espectral escolhida, orientações de como usar e como conservar o material, um informativo aos pais e/ou responsáveis 
sobre estresse visual, e uma orientação aos professores. Assim, a intervenção consistiu do uso por três meses em casa e na escola das lâminas espectrais para leitura de materiais impressos (ex., livros, revistas), ou sobre a tela do computador. No pósteste, os participantes $(n=57)$ incluídos no estudo de intervenção foram resubmetidos ao TELCS e ao RRT.

Como parte do objetivo científico deste trabalho, realizamos ainda uma reunião com os pais e professores dos participantes identificados com estresse visual, e que obtiveram resultados positivos frente ao tratamento proposto pela pesquisa. Orientou-se novamente sobre a condição, ressaltando que estas crianças são capazes, mas apresentam específica dificuldade de aprender a ler. Orientou-se também sobre o uso das lâminas espectrais para diminuir o desconforto na leitura e aumentar as habilidades cognitivas no processo de aprendizagem. Esclareceu-se que as lâminas espectrais são ferramentas não invasivas, de fácil portabilidade, sem efeitos colaterais e que não comprometem outras habilidades da criança requeridas na aprendizagem.

Em relação ao suporte pedagógico propriamente dito, foram sugeridas as seguintes estratégias para a escola, as quais poderiam ajudar na melhora do processo de aprendizagem: a) utilizar papel reciclável ou, idealmente, na tonalidade semelhante à(s) lâmina(s) espectral(is) específica(s) da criança para a impressão das atividades pedagógicas propostas; b) trazer o aluno para perto do quadro e da mesa do professor; c) reduzir o número de estímulos visuais em sala de aula, particularmente aqueles com contraste branco/preto; d) no caso de salas de aula próximos a quadra, evitar conteúdos novos quando a mesma estiver sendo usada, devido ao excesso de barulho; e) não submeter o aluno com estresse visual a atividades cronometradas ou competição com os colegas; f) utilizar giz ou caneta colorida para sublinhar trechos no quadro, pois isto facilita a cópia e evita a dispersão das crianças.

\subsection{Análise dos dados}

Os dados foram submetidos a análises de estatísticas descritivas (média, frequência simples e percentagem). A análise de variância com medidas repetidas (One-way Repeated Measures ANOVA) analisou a diferença pré-intervenção versus pósintervenção nos testes TELCS e RRT. Para estabelecer a significância clínica das diferenças, o $d$ de Cohen de 0.2 representa tamanho pequeno de efeito, 0.5 para um efeito médio e 0.8 para um efeito grande. $\mathrm{O}$ valor de $p$ inferior a 0.05 foi considerado estatisticamente significante.

\section{Resultados}

De acordo com o proposto, os participantes foram inicialmente avaliados quanto à sua habilidade de compreensão de leitura, sendo selecionadas aqueles com dificuldade de leitura para a idade (Percentil 7 a 30) (Tabela 1). Dos 237 participantes da amostra populacional selecionada, avaliados por meio do TELCS, 87 (37\%) foram classificados com dificuldade de compreensão de leitura, dos quais 5 (2\%) tinham erros refrativos não corrigidos. Não foram encontrados nesta amostra estudantes analfabetos (percentil inferior a 7 no TELCS). 
Tabela 1. Características da amostra quanto ao tamanho amostral, sexo, idade, escolaridade, perfil socioeconômico, desempenho de leitura e prevalência de estresse visual.

\begin{tabular}{|c|c|c|c|c|}
\hline \multicolumn{3}{|c|}{ CARACTERÍSTICAS } & \multirow{2}{*}{$\begin{array}{c}n \\
770\end{array}$} & \multirow{2}{*}{$\%$} \\
\hline \multirow{5}{*}{\multicolumn{2}{|c|}{ TAMANHO AMOSTRAL }} & Identificação inicial & & \\
\hline & & Avaliação da leitura & 237 & - \\
\hline & & Exame Oftalmológico & 87 & - \\
\hline & & Elegível no estudo de prevalência & 58 & 100 \\
\hline & & Incluída no estudo de intervenção & 57 & 98 \\
\hline \multirow{2}{*}{\multicolumn{2}{|c|}{ SEXO }} & Feminino & 36 & 62 \\
\hline & & Masculino & 22 & 38 \\
\hline \multirow{2}{*}{\multicolumn{2}{|c|}{ IDADE }} & Anos (Média \pm DP) & \multirow{2}{*}{\multicolumn{2}{|c|}{$\begin{array}{c}8.7 \pm 0.6 \\
7-10\end{array}$}} \\
\hline & & Amplitude em anos (Min.-Max.) & & \\
\hline \multirow{2}{*}{\multicolumn{2}{|c|}{ ESCOLARIDADE }} & $3^{0}$ ano & 19 & 33 \\
\hline & & $4^{0}$ ano & 39 & 67 \\
\hline \multirow{6}{*}{\multicolumn{2}{|c|}{ PERFIL SOCIOECONÔMICO }} & A2 & 1 & 2 \\
\hline & & B1 & 3 & 5 \\
\hline & & B2 & 13 & 22 \\
\hline & & $\mathrm{C} 1$ & 26 & 45 \\
\hline & & $\mathrm{C} 2$ & 10 & 17 \\
\hline & & $\mathrm{D}$ & 4 & 7 \\
\hline \multirow{5}{*}{ TELCS } & \multirow{5}{*}{$\begin{array}{l}\text { Compreensão de } \\
\text { sentenças }\end{array}$} & Percentil $\leq 7$ & 0 & 0 \\
\hline & & Percentil 10 & 18 & 31 \\
\hline & & Percentil 15 & 13 & 22 \\
\hline & & Percentil 25 & 9 & 16 \\
\hline & & Percentil 30 & 18 & 31 \\
\hline \multirow{4}{*}{ IRPS } & \multirow{4}{*}{$\begin{array}{l}\text { Seção } 1 \text { - Sintomas } \\
\text { de Estresse visual }\end{array}$} & Ausente & 0 & 0 \\
\hline & & Leve & 6 & 10 \\
\hline & & Moderado & 28 & 48 \\
\hline & & Severo & 24 & 41 \\
\hline \multirow{3}{*}{ RRT } & \multirow{3}{*}{$\begin{array}{l}\text { Ganho na taxa de } \\
\text { leitura com overlay }\end{array}$} & $\geq 5 \%$ & 27 & 47 \\
\hline & & $\geq 10 \%$ & 17 & 29 \\
\hline & & $\geq 15 \%$ & 10 & 17 \\
\hline SÍNTESE & IRPS e RRT & Prevalência de estresse visual severo & 7 & 12 \\
\hline
\end{tabular}

IRPS: Irlen Perceptual Reading Scale; TELCS: Teste de Leitura: Compreensão de Sentenças; RRT: Rate of Reading Test (Teste de Taxa de Leitura). Fonte: Autores (2021).

Após as etapas de seleção e elegibilidade da amostra, um total de 58 participantes com dificuldade de leitura e visão normal atenderam os critérios de inclusão do estudo de prevalência, sendo $62 \%$ do sexo feminino, $38 \%$ do sexo masculino, $67 \%$ do $4^{\circ}$ ano e $33 \%$ do $3^{\circ}$ ano do ensino fundamental (Tabela 1). A maior parte da amostra (62\%) foi composta por crianças que apresentavam 9 anos de idade no momento da coleta de dados (média $=8.7$ anos; Desvio Padrão $=0.6$ ). Como fonte de evidência baseada na estrutura interna do TELCS, a compreensão de leitura apresentou efeito de escolaridade significante $\left(3^{\circ}<\right.$ $4^{\circ}$ ano; $\left.F_{(1,57)}=9.4 ; p=0.003 ; d=0.91\right)$.

A maioria dos participantes pertence ao perfil socioeconômico C1 (45\%), seguida em frequência pelos perfis B2 (22\%), C2 (17\%), D (7\%), B1 (5\%) e A2 (2\%) (Tabela 1). Dos 58 participantes com dificuldade de leitura, avaliados pelos questionários de dificuldade e desconforto para a leitura (seção 1 do IRPS), $41 \%$ foram classificados com sintomas de estresse visual severos, $48 \%$ moderados e $10 \%$ leves, sem nenhum participante assintomático (Tabela 1).

Ainda segundo a Tabela 1 , no que diz respeito aos resultados obtidos pela aplicação do RRT na condição préintervenção, 47\%, 29\% e 17\% dos participantes apresentaram, com o uso das lâminas espectrais, ganhos na taxa de leitura de pelos menos $\geq 5 \%, \geq 10 \%$ e $\geq 15 \%$, respectivamente. Dessa forma, o estresse visual (de leve a severo) foi rastreado em $47 \%$ das crianças com dificuldade de leitura. Ao considerarmos, em conjunto, os resultados da Seção 1 do IRPS (apenas sintomas 
severos) e do RRT (aumento igual ou superior a $\geq 15 \%$ na taxa de leitura com o uso da lâmina espectral), o presente estudo encontrou uma prevalência de $12 \%$ de estresse visual severo em participantes com dificuldade de leitura.

Ao compararmos as condições pré- e pós-intervenção de três meses com as lâminas espectrais (Tabela 2), os participantes com dificuldade de leitura apresentaram forte melhora da compreensão de sentenças no TELCS, passando de um percentil médio de 19 para $35\left(F_{(1,56)}=50.5 ; p<0.0001 ; d=1.04\right)$. Com relação ao RRT, houve um significante e moderado aumento da taxa de leitura no pós-intervenção, passando de 70 para 81 palavras lidas em média por minuto $\left(F_{(1,56)}=69.7 ; p<\right.$ $0.0001 ; d=0.56)$.

Tabela 2. Desempenho de leitura dos participantes nas condições de pré- e pós-intervenção.

\begin{tabular}{|c|c|c|c|c|c|c|}
\hline & Variáveis & & Média \pm DP & $\overline{F_{(1,56)}}$ & $p$ & $d$ \\
\hline \multirow{4}{*}{ TELCS } & \multirow{4}{*}{ Compreensão de sentenças } & Bruto Pré & $10.9 \pm 2.9$ & \multirow{2}{*}{55.0} & \multirow{2}{*}{$<0.0001$} & \multirow{2}{*}{1.06} \\
\hline & & Bruto Pós & $15.0 \pm 4.6$ & & & \\
\hline & & Percentil Pré & $19.7 \pm 8.5$ & \multirow{2}{*}{50.5} & \multirow{2}{*}{$<0.0001$} & \multirow{2}{*}{1.04} \\
\hline & & Percentil Pós & $35.2 \pm 19.2$ & & & \\
\hline \multirow{2}{*}{ RRT } & \multirow{2}{*}{ Taxa de Leitura } & Bruto Pré (ppm) & $70.3 \pm 20.6$ & \multirow{2}{*}{69.7} & \multirow{2}{*}{$<0.0001$} & \multirow{2}{*}{0.56} \\
\hline & & Bruto Pós (ppm) & $81.9 \pm 20.9$ & & & \\
\hline
\end{tabular}

TELCS: Teste de Leitura: Compreensão de Sentenças. RRT: Rate of Reading Test (Teste de Taxa de Leitura). ppm: palavras por minuto. Fonte: Autores (2021).

\section{Discussão}

A amostra elegível para compor esta pesquisa foi de 58 alunos com dificuldade de leitura, todos do $3^{\circ}$ e do $4^{\circ}$ anos do ensino fundamental, fase esta que a criança consolida a sua habilidade de leitura, como resultado da neuroplasticidade de diversas vias neuronais envolvidas no processo de aprendizagem (Faria, 2011). Em uma fase inicial de desenvolvimento do projeto, os participantes selecionados foram submetidos ao TELCS, teste psicométrico padrão ouro com normas para avaliar a compreensão de leitura (Vilhena \& Pinheiro, 2020), sendo 37\% classificados com dificuldade de leitura (Percentil igual ou inferior a 30).

Nesse sentido, o Programa Internacional de Avaliação de Estudantes (Pisa), que avalia o desempenho de jovens na faixa etária dos 15 anos em leitura, matemática e ciências revelou que 50\% dos estudantes brasileiros não atingiram o mínimo de proficiência em leitura, levando o Brasil a ocupar a faixa entre $55^{\circ}$ e $59^{\circ}$ colocado (INEP, 2019). Esse resultado é bastante preocupante, tendo em vista que as dificuldades de leitura impedem que os estudantes avancem satisfatoriamente nos estudos, contribuindo para elevadas taxas de abandono escolar.

De fato, o Brasil tem a terceira maior taxa de evasão entre os 100 países com maior Índice de Desenvolvimento Humano, com um índice de 24,3\%, só atrás da Bósnia Herzegovina (26,8\%) e das ilhas de São Cristovam e Névis no Caribe (26,5\%) (Silva Filho \& Araújo, 2017). Quando chegam ao ensino superior, estes estudantes não dominam a leitura e a escrita, competências fundamentais para a compreensão de textos, o que dificulta e posterga sua permanência na graduação (Auriglietti, 2014; Silva Filho \& Araújo, 2017). Segundo Leite e Cadei (2016), 29\% dos alunos que chegam ao ensino superior não apresentam essas competências desenvolvidas.

Passando-se ao rastreio do estresse visual (seção 1 do IRPS), observamos que 24 participantes com dificuldade de leitura, correspondendo a $41 \%$ da amostra do presente estudo, reportaram nível de estresse severo. O mesmo nível de estresse visual foi verificado em 16\% de uma amostra populacional de estudantes de São Paulo/Brasil (Garcia, Momensohn-Santos \& Vilhena, 2017) e em 26\% dos alunos de um estudo desenvolvido em Cuenca/Equador (Bernal, 2015). De acordo com os 
presentes dados, $90 \%$ dos participantes com dificuldade de leitura apresentaram queixas de estresse visual entre moderado a severo, com os demais $10 \%$ relatando sintomas leves.

Ao utilizar o critério de corte mais leniente no RRT para rastrear o estresse visual (leve a severo), foi verificado que $47 \%$ dos participantes apresentaram melhora de pelo menos $\geq 5 \%$ na taxa de leitura com o uso das lâminas espectrais. Uma revisão sistemática com meta-análise verificou que o estresse visual (de leve a severo), com o mesmo critério de $\geq 5 \%$ no RRT, foi identificado em $33 \%$ da população geral, $38 \%$ de crianças com dificuldade de leitura, $61 \%$ de adultos com dificuldade de leitura, 74\% no Transtorno do Espectro Autista, 77\% na esclerose múltipla, e 92\% na síndrome de Tourette (Vilhena, Guimarães \& Guimarães, 2019). Ao se utilizar o critério de corte mais rigoroso de $\geq 15 \%$ no RRT, que representa uma melhoria da taxa de leitura com o overlay para além do intervalo de variabilidade intra-individual (Garcia, Momensohn-Santos \& Vilhena, 2017), foi encontrada a prevalência de $17 \%$ de estresse visual severo.

Considerando, em conjunto, os resultados do IRPS com o RRT, foi encontrada uma prevalência final de $12 \%$ de estresse visual severo entre os participantes com dificuldade de leitura, o que se configura como fator de risco grave para dificuldades funcionais e acadêmicas. Esta proporção é semelhante àquela encontrada em crianças com dificuldade de leitura (19\%) em estudo prévio realizado em Minas Gerais (Faria, 2011).

Como detalhado na parte metodológica, a tonalidade das lâminas ou overlays é escolhida de maneira idiossincrática, específica e consistente. O desempenho de leitura com as lâminas ideais é superior a quando elas são escolhidas de forma aleatória (Bouldoukian, Wilkins \& Evans, 2002; Wilkins \& Lewis, 1999) ou por opção estética (Ludlow, Wilkins \& Heaton, 2008), falando a favor da especificidade da intervenção. A leitura é a habilidade cognitiva que mais responde às overlays, as quais são usadas para melhorar o reconhecimento das letras, a taxa de leitura e a compreensão do texto, na medida em que melhoram o contraste e diminuem o brilho do papel (Noble et al., 2004; Guimarães \& Guimarães, 2012; Vilhena, Guimarães \& Guimarães, 2019; Wilkins \& Evans, 2010).

Conforme demonstrado, os participantes melhoraram a compreensão de sentenças no TELCS $(p<0.0001 ; d=1.06) \mathrm{e}$ a taxa de leitura no RRT ( $p<0.0001 ; d=0.56$ ), quando comparados com a linha de base da pré-intervenção (Tabela 2 ). A título de conhecimento, um aumento de $38 \%$ no percentil do TELCS equivale a quatro sentenças lidas a mais a cada cinco minutos, enquanto que um aumento de $17 \%$ na taxa de leitura no RRT equivale a doze palavras lidas a mais por minuto.

É pacífico o entendimento de que os avanços em Neurociências impactam decisivamente na condução do processo educativo, ajudando a identificação e a superação das dificuldades enfrentadas diariamente pelos profissionais de educação (Damasceno et al., 2021). O Déficit Magnocelular e a Hiperexcitabilidade Cortical são as duas principais teorias sobre a etiologia do estresse visual associado à dificuldade de leitura. Na primeira, propõe-se que parte da dificuldade de leitura possui como causa um déficit na via magnocelular retino-geniculo-cortical, com prejuízo do processamento temporal, percepção de movimento, controle do movimento ocular e da atenção visual (Flint \& Pammer, 2019; Garcia, Vilhena, Guimarães, Pinheiro \& Momensohn-Santos, 2019; Stein, 2001; Vilhena, Guimarães, Guimarães \& Pinheiro, 2021). Já pela teoria da Hiperexcitabilidade Cortical é proposto que uma estimulação cortical intensa, como a que ocorre durante a leitura prolongada, poderia reduzir os mecanismos de inibição do córtex visual, provocando uma estimulação excessiva dos neurônios corticais e causando as distorções visuoperceptuais (Allen, Gilchrist \& Hollis, 2008; Chouinard et al., 2012; Wilkins \& Evans, 2010). Enquanto que na primeira teoria a intervenção com o uso das lâminas espectrais regularizaria a transmissão do impulso pela via magnocelular, na segunda, as overlays redistribuiriam a atividade visual para neurônios corticais com menor excitabilidade, evitando as áreas de hiperexcitação.

Embora existam críticas em relação à tentativa de explicação biológica e crescente medicalização de atitudes como agitação e desatenção em crianças (Silva \& Batista, 2020), as dificuldades de aprendizagem estão cada vez mais prevalentes, exigindo dos profissionais de educação habilidades de identificar e diminuir as limitações de seus alunos (Loew, Alba \& 
Watson, 2013; Peng, 2013; Rotta, Ohlweiler \& Riesgo 2016). Por óbvio, a existência de fatores biológicos envolvidos não minimiza os impactos de outros determinantes sociais e psicológicos envolvidos no processo de aprendizagem, sempre objetivando uma abordagem integrativa.

\section{Considerações Finais}

Em conclusão, o presente estudo demonstrou prevalência de 12\% de estresse visual (sintomas severos) na amostra estudada, sendo que os participantes em uso contínuo das overlays por três meses apresentaram um forte e significante aumento do desempenho de leitura, avaliado pelo TELCS e RRT, entre as condições de linha de base e pós-intervenção.

Assim, destacamos o uso das overlays como método de intervenção de alta eficiência, baixo custo e simples manejo para tratamento do estresse visual associado à dificuldade de leitura. A alta relação custo-benefício é particularmente importante quando se avalia o atual cenário político e econômico brasileiro, bem como as condições do processo educativo no país. Assim, esta pesquisa procurou contribuir para a difusão do conhecimento sobre a síndrome no contexto científico, na medida em que atinge profissionais interdisciplinarmente nas áreas da saúde e da educação, abrindo um horizonte de informações e qualificando melhor estes profissionais para os desafios que irão encontrar dentro de seus consultórios ou em salas de aula. No contexto social, esta pesquisa pretendeu contribuir para a valorização da capacidade dos próprios estudantes, os quais, sem o diagnóstico adequado, acabam discriminados pelos próprios professores ou pelos colegas de sala, na medida em que os ajudou a desenvolver seu aprendizado, e mais importante, a resgatar a sua autoestima.

Uma vantagem da amostra utilizada pelo presente estudo é a não inclusão de participantes com diagnóstico de erros refracionais não corrigidos, uma vez que podem comprometer a acuidade visual, a leitura e a identificação do estresse visual. Uma limitação do estudo foi a ausência do grupo controle, pois todos os participantes passaram pela intervenção com as lâminas espectrais durante os três meses de pesquisa. Desta forma, sugerimos que próximos estudos a serem desenvolvidos neste tema considerem a inclusão de grupos sem intervenção, de forma a evidenciar ainda mais os benefícios da estratégia. Ademais, como a amostra do presente estudo foi restrita àqueles alunos com dificuldade de leitura, sugerimos que a intervenção seja testada também em leitores proficientes, uma vez que o estresse visual pode igualmente afetá-los.

\section{Referências}

Allen, P. M., Gilchrist, J. M., \& Hollis, J. (2008). Use of Visual Search in the Assessment of Pattern-Related Visual Stress (PRVS) and Its Alleviation by Colored Filters. Investigative Ophthalmology \& Visual Science, 49(9), 4210-4218. https://doi.org/10.1167/iovs.07-1587

Alves, M. R., \& Kara-José, N. (1998). Campanha “Veja Bem Brasil”. Manual de Orientação. Conselho Brasileiro de Oftalmologia.

Alves, M. T. G., Soares, J. F., \& Xavier, F. P. (2014). Índice socioeconômico das escolas de educação básica brasileiras. In: Ensaio: Avaliação e Políticas Públicas em Educação, 22, 671-703.

Alves, P. C., Melo, T. C. L. C., Santos, J. L. R. dos, Machado, R. R., Rocha, F. H., Vasconcelos, F. H. P. de, Linhares, A. E. P., Ferreira, F. V., \& Félix, T. A., (2021). Psychology and school failure/complaint: an analysis of scientific production. Research, Society and Development, 10(10), e187101018810. https://doi.org/10.33448/rsd-v10i10.18810

Associação Brasileira de Empresas de Pesquisa (ABEP). (2016). Critério de Classificação Econômica Brasil.

Auriglietti, R. C. R. (2014). Evasão e abandono escolar: causas, consequências e alternativas - o combate à evasão escolar sob a perspectiva dos alunos. Cadernos PDE, 1, 1-21.

Bernal, M. (2015). Prevalencia del síndrome Meares-Irlen/Estrés Visual que afecta la lectura en niños de tercer grado. Maskana, 6(1 SE-Artículos científicos). https://doi.org/10.18537/mskn.06.01.06

Bicalho, L. F., Almeida, M. Z. T. de, Guimarães, M. R., Silva, J. R. G., \& Fully, F. (2015). Síndrome de Irlen: Um Olhar Atento sobre o Funcionamento Cerebral Durante a Leitura. Acta Biomedica Brasiliensia, 6(1), 35-44.

Bouldoukian, J., Wilkins, A. J., \& Evans, B. J. W. (2002). Randomised controlled trial of the effect of coloured overlays on the rate of reading of people with specific learning difficulties. Ophthalmic and Physiological Optics, 22(1), 55-60. https://doi.org/https://doi.org/10.1046/j.1475-1313.2002.00002.x 
Boyle, C., \& Jindal-Snape, D. (2012). Visual-perceptual difficulties and the impact on children's learning: are teachers missing the page? Support for Learning, 27(4), 166-171. https://doi.org/https://doi.org/10.1111/1467-9604.12001

Chouinard, B. D., Zhou, C. I., Hrybouski, S., Kim, E. S., \& Cummine, J. (2012). A Functional Neuroimaging Case Study of Meares-Irlen Syndrome/Visual Stress (MISViS). Brain Topography, 25(3), 293-307. https://doi.org/10.1007/s10548-011-0212-z

Coltheart, M., Rastle, K., Perry, C., Langdon, R., \& Ziegler, J. (2001). DRC: A dual route cascaded model of visual word recognition and reading aloud. In Psychological Review, 108(1), 204-256. https://doi.org/10.1037/0033-295X.108.1.204

Costa, A. M. (2014). Síndrome de Meares-Irlen e as alterações na leitura. Anais I Congresso Internacional de Educação e Inclusão. https://www.editorarealize.com.br/artigo/visualizar/9232

Damasceno, G. C., Cabral, D. A. C., Campos, F. M. S., Medeiros, J. P. do V., Cabral, L. G. C., \& Cabral, K. M. C. (2021). Neuroscience contributions to pedagogy: a necessary dialogue. Research, Society and Development, 10(1), e33710111846. https://doi.org/10.33448/rsd-v10i1.11846

Estrela, C. (2018). Metodologia Científica: Ciência, Ensino, Pesquisa. Editora Artes Médicas.

Dehaene, S. (2012). Os neurônios da leitura - Como a Ciência Explica a Nossa Capacidade de Ler. Penso.

Evans, B. J. W., Busby, A., Jeanes, R., \& Wilkins, A. J. (1995). Optometric correlates of Meares-Irlen Syndrome: a matched group study. Ophthalmic and Physiological Optics, 15(5), 481-487. https://doi.org/https://doi.org/10.1016/0275-5408(95)00063-J

Evans, B. J. W., Wilkins, A. J., Brown, J., Busby, A., Wingfield, A., Jeanes, R., \& Bald, J. (1996). A preliminary investigation into the aetiology of MearesIrlen syndrome. Ophthalmic and Physiological Optics, 16(4), 286-296. https://doi.org/10.1016/0275-5408(95)00119-0

Faria, L. N. (2011). Frequência da Síndrome de Meares- Irlen entre Alunos com Dificuldades de Leitura Observadas no Contexto Escolar. [Instituto de Ciências Biológicas da Universidade Federal de Minas Gerais]. https://repositorio.ufmg.br/bitstream/1843/BUOS-962H8G/1/pdf_disserta_o_laura_ver sao_final_18_01_2012_revisto_leonor_0_.pdf

Ferreira, A. P. A., Albuquerque, R. C., Rabelo, A. R. de M., Farias, F. C. de, Correia, R. C. de B., Gagliardo, H. G. R. G., \& Lima, A. C. V. M. de S. (2011). Comportamento visual e desenvolvimento motor de recém-nascidos prematuros no primeiro mês de vida. In: Journal of Human Growth and Development, 21 , 335-343).

Flint, S., Pammer, K. (2019). It is the egg, not the chicken; dorsal visual deficits present in dyslexia are not present in illiterate adults. Dyslexia, 25(1), 69-83. https://doi.org/10.1002/dys.1607

Garcia, A. C. O., Momensohn-Santos, T. M., \& Vilhena, D. A. (2017). Effects of Spectral Overlays on Reading Performance of Brazilian Elementary School Children. Folia Phoniatrica et Logopaedica, 69(5-6), 219-225. https://doi.org/10.1159/000484139

Garcia, A. C. O., Vilhena, D. A., Guimarães, M. R., Pinheiro, Ângela M. V., \& Momensohn-Santos, T. M. (2019). Association between auditory temporal and visual processing in reading skill. Revista CEFAC, 21(5), e6119. https://dx.doi.org/10.1590/1982-0216/20192156119

Graziano, R. M., \& Leone, C. R. (2005). Problemas oftalmológicos mais freqüentes e desenvolvimento visual do pré-termo extremo. Jornal de Pediatria, 81, S95-S100).

Guimarães, M. R., \& Guimarães, I. R. (2013). Aprendizagem e Leitura - Síndrome de Irlen. In Neurociência e Educação, 350. Book Editora.

Guimarães, M. R., \& Guimarães, R. (2012). Avaliação da visão Funcional em Disturbios de Aprendizagem - O Papel do Oftalmologista nos Déficits de Visão e Aprendizagem. In Dislexia e Distúrbios de Aprendizagem, 159-174. Cultura Médica.

Instituto Nacional de Estudos e Pesquisas Educacionais Anísio Teixeira (INEP). (2019). Pisa 2018 revela baixo desempenho escolar em leitura, matemática e ciências no Brasil.

Irlen, H. (2003). Reading Perceptual Scale. Perceptual Development Corporation.

Kamakura, W., \& Mazzon, J. A. (2016). Critérios de estratificação e comparação de classificadores socioeconômicos no Brasil. In: Revista de Administração de Empresa, 56, 55-70.

Kruk, R., Sumbler, K., \& Willows, D. (2008). Visual processing characteristics of children with Meares-Irlen syndrome. Ophthalmic and Physiological Optics, 28(1), 35-46. https://doi.org/https://doi.org/10.1111/j.1475-1313.2007.00532.x

Lamônica, D. A. C., Carlino, F. C., \& Alvarenga, K. de F. (2010). Avaliação da função auditiva receptiva, expressiva e visual em crianças prematuras. In PróFono Revista de Atualização Científica, 22, 19-24).

Leite, F. R., \& Cadei, M. M. S. (2016). Analfabetismo funcional: Uma realidade preocupante. Revista Científica Do Instituto Ideia, 1, 13-19.

Loew, S. J., Alba, M. E. F., \& Watson, K. (2013). Incidence of Meares-Irlen/ Visual Stress Syndrome in Reading and Learning Disorders: Does Fluorescent Lighting in Classrooms affect Literacy and Numeracy? Aula Abierta, 41(3), 23-32.

Ludlow, A. K., Wilkins, A. J., \& Heaton, P. (2008). Colored overlays enhance visual perceptual performance in children with autism spectrum disorders. Research in Autism Spectrum Disorders, 2(3), 498-515. https://doi.org/https://doi.org/10.1016/j.rasd.2007.10.001

Machado, M. S. M., \& Maluf, M. R. (2019). Como evolui a compreensão da leitura em alunos do ensino fundamental [How reading comprehension evolves in elementary school students]. Psicologia da Educação, (49), 57-66. https://doi.org/10.5935/2175-3520.20190019 
Medina, G. B. K., \& Guimarães, S. R. K. (2019). Leitura de Estudantes com Dislexia do Desenvolvimento: Impactos de uma Intervenção com Método Fônico Associado à Estimulação de Funções Executivas. Revista Brasileira de Educação Especial, 25(1), 155-174. http://dx.doi.org/10.1590/s141365382519000100010

Medina, G. B. K., \& Guimarães, S. R. K. (2021). Reading in developmental dyslexia: the role of phonemic awareness and executive functions. Estudos de Psicologia (Campinas), 38, e180178. https://doi.org/10.1590/1982-0275202138e180178

Medina, G. B. K., Souza, F. F., \& Guimarães, S. R. K. (2018). Funções executivas e leitura em crianças brasileiras com dislexia do desenvolvimento [Executive functions and reading in Brazilian children with developmental dyslexia]. Revista Psicopedagogia, 35(107), 168-179.

Nandakumar, K., \& Leat, S. J. (2008). Dyslexia: a review of two theories. Clinical and Experimental Optometry, 91(4), 333-340. https://doi.org/https://doi.org/10.1111/j.1444-0938.2008.00277.x

Noble, J., Orton, M., Irlen, S., \& Robinson, G. (2004). A controlled field study of the use of coloured overlays on reading achievement. Australian Journal of Learning Disabilities, 9(2), 14-22. https://doi.org/10.1080/19404150409546760

Oliveira, A. L. S. de, Kaiser, V., Azambuja, T. de O., Mallmann, L. U., Lukrafka, J. L., \& Reppold, C. T. (2016). Visual-Motor Maturity and Executive Functions in Schoolchildren. In Paidéia (Ribeirão Preto), 26, 215-223).

Peng, Y. T. (2013). Dyslexia and Vision - A review of current evidence and clinical interventions. Medical Grapevine.

Piccolo, L. da R., \& Salles, J. F. de. (2013). Vocabulário e memória de trabalho predizem desempenho em Leitura de crianças. Revista Psicologia: Teoria e Prática, 15(2), 180-191.

Pinheiro, Â. M. V., Vilhena, D. A., \& Santos, M. A. C. (2017). PROLEC-T - Prova de Compreensão de Texto: Análise de suas Características Psicométricas [PROLEC-T - Text comprehension test: psychometric properties analysis]. Temas em Psicologia [Trends in Psychology], 25(3). http://dx.doi.org/10.9788/tp2017.3-08.

Rotta, N. T., Ohlweiler, L., \& Riesgo, R. dos S. (2016). Transtornos da Aprendizagem: Abordagem Neurobiológica e Multidisciplinar. (2a ed.). Artmed.

Salles, J. F. de. (2005). Habilidades e Dificuldades de Leitura e Escrita em Crianças de $2^{a}$ série: Abordagem neuropsicológica cognitiva. [Universidade Federal do Rio Grande do Sul]. https://www.lume.ufrgs.br/bitstream/handle/10183/4197/000453537.pdf?sequence=1

Schirmer, C. R., Fontoura, D. R., \& Nunes, M. L. (2004). Distúrbios da aquisição da linguagem e da aprendizagem. In Jornal de Pediatria, 80, $95-103$.

Silva Filho, R. B., \& Araújo, R. M. de L. (2017). Evasão e abandono escolar na educação básica no Brasil: fatores, causas e possíveis consequências. Educação Por Escrito, 8(1 SE-Artigos), 35-48. https://doi.org/10.15448/2179-8435.2017.1.24527

Silva, I. P. D. da, \& Batista, C. G. (2020). Crianças agitadas/desatentas: modelos de explicação. In Pro-Posições, 31.

Siqueira, C. M., \& Gurgel-Giannetti, J. (2011). Mau desempenho escolar: uma visão atual. In Revista da Associação Médica Brasileira, $57,78-87$.

Souza, J. R. de, \& Silva, A. de O. V. da. (2021). Factors that interfere in the teaching and learning process. Research, Society and Development, 10(6), e29210616071. https://doi.org/10.33448/rsd-v10i6.16071

Stein, J. (2001). The magnocellular theory of developmental dyslexia. Dyslexia, 7(1), 12-36. https://doi.org/https://doi.org/10.1002/dys.186

Toledo, C. C., Paiva, A. P. G., Camilo, G. B., Maior, M. R. S., Leite, I. C. G., \& Guerra, M. R. (2010). Detecção precoce de deficiência visual e sua relação com o rendimento escolar: In Revista da Associação Médica Brasileira, 56, 415-419.

Uccula, A., Enna, M., \& Mulatti, C. (2014). Colors, colored overlays, and reading skills. In Frontiers in Psychology, 5, 833. https://www.frontiersin.org/article/10.3389/fpsyg.2014.00833

Vilhena, D. A., \& Pinheiro, Â. M. V. (2020). Reliability, Validity and Standardization of the Reading Test: Sentence Comprehension. Psicologia: Teoria e Pesquisa, 36, e36325. https://dx.doi.org/10.1590/0102.3772e36325

Vilhena, D. A., Guimarães, M. R., Guimarães, R. Q., \& Pinheiro, A. M. V. (2018). Tratamento do Estresse Visual na leitura: características físicas e mecanismos neurais de ação das lâminas espectrais (overlays). Paidéia, 19(1), 83-97.

Vilhena, D. A., Guimarães, M. R., Guimarães, R. Q., \& Pinheiro, Â. M. V. (2019). Propriedades Psicométricas do Teste de Taxa de Leitura: validade de conteúdo e fidedignidade [Psychometric Properties of the Rate of Reading Test: content validity and reliability]. Anais do Encontro Mineiro de Neuropsicologia Escolar. Universidade Federal de Minas Gerais.

Vilhena, D. A., Guimarães, M. R., Guimarães, R. Q., \& Pinheiro, Â. M. V. (2021). Magnocellular visual function in developmental dyslexia: deficit in frequency-doubling perimetry and ocular motor skills. Arq Bras Oftalmol, 84(5).

Vilhena, D. A., Sucena, A., Castro, S. L., \& Pinheiro, Â. M. V. (2016). Reading Test-Sentence Comprehension: An Adapted Version of Lobrot's Lecture 3 Test for Brazilian Portuguese. Dyslexia, 22(1), 47-63. https://doi.org/https://doi.org/10.1002/dys.1521

Vilhena, D. de A., Guimarães, M. R., \& Guimarães, R. Q. (2019). Melhora do desempenho de leitura com o uso de lâminas espectrais: revisão sistemática e meta-análise. Psicologia Argumento; 36 (93). https://doi.org/10.7213/Psicolargum.36.93.AO05

Wilkins, A. J., \& Evans, B. J. W. (2010). Visual stress, its treatment with spectral filters, and its relationship to visually induced motion sickness. Applied Ergonomics, 41(4), 509-515. https://doi.org/https://doi.org/10.1016/j.apergo.2009.01.011

Wilkins, A. J., Jeanes, R. J., Pumfrey, P. D., \& Laskier, M. (1996). Rate of Reading Test®: its reliability, and its validity in the assessment of the effects of coloured overlays. Ophthalmic and Physiological Optics, 16(6), 491-497. https://doi.org/10.1046/j.1475-1313.1996.96000282.x 
Research, Society and Development, v. 10, n. 11, e430101119914, 2021

(CC BY 4.0) | ISSN 2525-3409 | DOI: http://dx.doi.org/10.33448/rsd-v10i11.19914

Wilkins, A., \& Lewis, E. (1999). Coloured Overlays, Text, and Texture. Perception, 28(5), 641-650. https://doi.org/10.1068/p2761

Zucoloto, K. A., \& Sisto, F. F. (2002). Dificuldades de aprendizagem em escrita e compreensão em leitura. Interação Em Psicologia, 6(2), 157-166. http://dx.doi.org/10.5380/psi.v6i2.3303 